\title{
Verification of a distributed Cache Memory by using abstractions *
}

\author{
Susanne Graf \\ VERIMAG ${ }^{\star}$, Rue Lavoisier, F-38330 Monbonnot, e-mail: graf@imag.fr
}

\begin{abstract}
The purpose of this paper is to verify a distributed cache memory system by using the following general verification method: verify the properties characterizing a complex system on some small finite abstraction of it, obtained as a composition of abstractions of each component of the system. For a large class of systems including infinite state systems, the abstractions of the components can be obtained by replacing all operators on concrete domains by abstract operators on some abstract domain. This holds also for the abstraction of the control part of the system as we consider a kind of guarded command programs where all the control is expressed in terms of operations on explicit control variables.
\end{abstract}

\section{Introduction}

The purpose of this paper is to show the practical applicability of the verification method proposed in [LGS+92, GL93a, GL93b, CGL91, Lon93] for infinite state systems. This verification method, based on the principle of abstract interpretation [CC77], proposes to verify a program defining some complex system, where the specification must be given in the form of a set of $\forall \mathrm{CTL}^{*}$ [SG90] formulas, as follows: define an appropriate abstract program, obtained compositionally from the the given program, and verify the required properties on it. Our way of computing abstract programs is very similar to that proposed in [CGL91, Lon93], but our concept of compositionality is different from that proposed in [Lon93] or in [Pnu85]. We construct a global abstraction of the system by composing abstractions of its components, whereas the other method consists in deducing properties of the composed system from properties of its components. Both approaches are useful, but in the example we treat in this paper, the global properties cannot be deduced easily from properties of the components. An abstraction of each component is obtained applying the principle of abstract interpretation by means of a relation $\varrho$ relating the domain of its variables and the domain of the set of some abstract variables.

In [Loi94] is described a tool allowing to verify finite state systems in a fully automatic way by using this method. Here, we show that the same method is also tractable in practice for infinite state systems where a complete automatization is not possible. In fact, if depending on the formula one wants to verify - for each component $P_{i}$ one can guess an appropriate abstraction relation $\varrho_{i}$ verification becomes often a relatively simple task as

- the corresponding finite state abstract program is reasonably easy to obtain,

- the verification of the properties on the obtained abstract program can be done fully automatically.

In Section 2, we recall all the ingredients we need for our verification method:

- a simple program formalism similar to that used e. g., in [Pnu86],

\footnotetext{
* This work was partially supported by ESPRIT Basic Research Action "REACT"

** Verimag is a joint laboratory of CNRS, Institut National Polytechnique de Grenoble, Universitée J. Fourier and Verilog SA associated with IMAG
} 
- a method to compute abstract programs, consisting in defining for each operator on the concrete domains a corresponding abstract operator; this is the only step in the proposed method that cannot be fully automated.

- the temporal logic CTL* and its fragments, used for the description of properties,

- the preservation results allowing to deduce the validity of a property on the concrete program from its validity on the abstract program and

- the compositionality results allowing to compute an abstract program by composing abstractions of its components.

We illustrate all the definitions and results on a small buffer example. In Section 3, we verify - by applying in a systematic way our method - a distributed cache memory system defined in [ABM93]. In [DGJ+93] several (complex) correctness proofs are given for this system based on different methods. Using our method, the verification of this system is almost as simple as the verification of the tiny buffer, as we need almost the the same abstract operations.

\section{A verification method using abstractions}

\subsection{A program description formalism}

We adopt a simple program formalism which is not meant as a real programming language but which is sufficient to illustrate our method. A complex system is a parallel composition of basic programs $\mathbf{P}$ of the following form

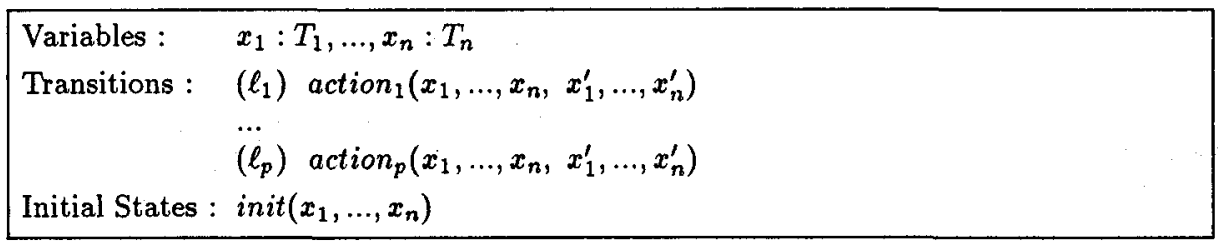

where $x_{i}$ are variables of type $T_{i}$ and $L_{P}=\left\{\ell_{1}, \ldots, \ell_{n}\right\}$ is the set of program labels. Each action $i$ is an expression with variables in the set of program variables and a set of primed variables which is a copy of the set of state variables; as in [Pnu86, Lam91], action represents a transition relation on the domain of the program variables by interpreting the valuations of $X_{P}=\left(x_{1}, \ldots, x_{n}\right)$ as the state before, and the valuations of $X_{P}^{\prime}=\left(x_{1}^{\prime}, \ldots, x_{n}^{\prime}\right)$ as the state after the transition. We denote the set of valuations of $X_{P}$ by $\operatorname{Val}\left(X_{P}\right)$.

Semantics : Program $P$ defines in an obvious manner a transition system $S_{P}=\left(Q_{P}, R_{P}\right)$ where

$-Q_{P}=\operatorname{Val}\left(X_{P}\right)$ is the set of states,

- $R_{P} \subseteq Q_{P} \times Q_{P}$ is a transition relation defined by $R_{P}=\left\{\left(q, q^{\prime}\right) \mid \exists i\right.$. $\left.\operatorname{action}_{i}\left(q, q^{\prime}\right)\right\}$.

The predicate init defines the set of initial states. It is used in the formulas specifying the program: properties are in general of the form init $\Rightarrow \phi$ where $\phi$ expresses the property one wants to verify.

We do not distinguish variables representing inputs as they need not be treated in a particular manner. However, we annotate in the programs the variables which are meant as inputs as this makes programs easier to read.

Labels are used to name "events" or "actions". If $\ell_{i}$ is a label and $\left(v, v^{\prime}\right)$ a pair of valuations such that $\operatorname{action}_{i}\left(v, v^{\prime}\right)$ is true, then the transition from state $v$ to state $v^{\prime}$ is 
an event $\ell$. If $e$ is the valuation of the "input" variables extracted from $v$, then we call this event also $\ell_{i}(e)$. Events are used for the expression of properties.

Example 1 an infinite lossy buffer. The following program represents an unbounded buffer taking as input elements $e$ of some data domain elem. The event push(e) enters $e$ (if it has never been entered yet) into the buffer or arbitrarily "loses" it, and pop(e) takes $e$ out of the buffer if it is its first element.

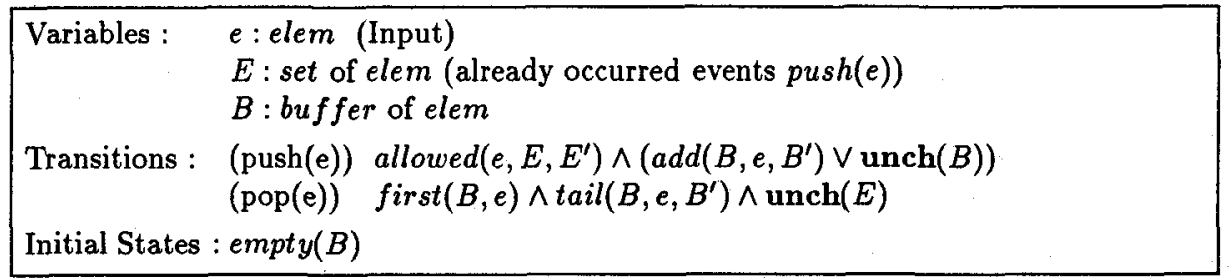

$E$ contains the elements $e$ such that push $(e)$ has already occurred, and allowed $\left(e, E, E^{\prime}\right)$ is necessarily false if $e \in E$. All other predicates have the intuitive meanings: $\operatorname{add}\left(B, e, B^{\prime}\right)$ holds if $B^{\prime}$ is obtained by adding element $e$ at the end of the buffer $B$; $\operatorname{tail}\left(B, e, B^{\prime}\right)$ holds if $B^{\prime}$ is obtained by eliminating $e$ from $B$ if $e$ is its first element (first $(B, e)$ is true); empty $(B)$ is true if $B$ is the empty buffer. unch $(X)$, where $X=x_{1}, \ldots x_{n}$ is a tuple of program variables, represents the transition relation which lets all variables in $X$ unchanged, i. e., $\operatorname{unch}(X)=\bigwedge_{i}\left(x_{i}^{\prime}=x_{i}\right)$.

We use predicates of the form $\operatorname{add}\left(B, e, B^{\prime}\right)$ instead of $B^{\prime}=A D D(B, e)$ where $A D D$ is a function, as abstract operations are in general nondeterministic. This is also the way of representing operations which is proposed, e. g. in [CGL91, Lam91].

Composed programs : In [GL93b] we obtain our results for more general parallel composition operators, but here we need only asynchronous composition. If $P_{1}$ and $P_{2}$ are programs defined on a tuple of state variables $X_{1}$, respectively $X_{2}$, then $P_{1} \| P_{2}$ is the parallel composition of $P_{1}$ and $P_{2}$ defining the transition system $S=\left(\operatorname{Val}\left(X_{1} \cup X_{2}\right), R\right)$ where

$$
R=R_{P_{1}} \wedge \operatorname{unch}\left(X_{2}-X_{1}\right) \vee R_{P_{2}} \wedge \operatorname{unch}\left(X_{1}-X_{2}\right)
$$

Each transition of $P_{1} \| P_{2}$ is either a transition of $P_{1}$ which leaves all variables which are declared in $P_{2}$ but not in $P_{1}$ unchanged or the other way round.

\subsection{Abstract programs}

As proposed in [CGL91, LGS +92 ], given a program Prog and a predicate $\varrho$ on the variables of Prog and a tuple of abstract variables $X^{A}=\left(x_{1}^{A}, \ldots x_{m}^{A}\right)$, representing a relation between the concrete and the abstract domain (a function in [CGL91]), then any program $\operatorname{Prog}^{A}$ defined on $X^{A}$, such that for each action action of Prog there exists an action action $^{A}$ of $\operatorname{Prog}^{A}$ with the same label, such that

$$
\begin{aligned}
& \exists X \exists X^{\prime} \cdot \varrho\left(X, X^{A}\right) \wedge \varrho\left(X^{\prime}, X^{A^{\prime}}\right) \wedge \operatorname{action}\left(X, X^{\prime}\right) \Rightarrow \operatorname{action}^{A}\left(X^{A}, X^{A^{\prime}}\right) \\
& \quad \text { and } \\
& \exists X \cdot \varrho\left(X, X^{A}\right) \wedge \text { init }(X) \Rightarrow \text { init }{ }^{A}\left(X^{A}\right) \\
& \text { is an abstraction or more precisely a } \varrho \text {-abstraction of Prog. }
\end{aligned}
$$

When verifying composed programs, it is interesting to compute an abstract program compositionally, i. e., by composing abstract component programs. From a more general result given in [GL93a], we obtain the following result which is sufficient for the verification of the distributed cache memory system in the following section. 
Proposition 1. Let $P_{1}$ and $P_{2}$ be programs and $\varrho_{i}$ total functions from the domain of the variables of $P_{i}$ into some abstract domains such that $\varrho_{1} \cap \varrho_{2}$ is total and $P_{1}^{A}, P_{2}^{A}$ are $\varrho_{i}$-abstractions of $P_{i}$, then $P_{1}^{A} \| P_{2}^{A}$ is a $\left(\varrho_{1} \cap \varrho_{2}\right)$-abstraction of $P_{1} \| P_{2}$.

Computation of abstract programs in practice : The idea of abstract interpretation [CC77] is to replace every function on the concrete domain used in the program by a corresponding abstract function on the abstract domain, and then to analyze the so obtained simpler abstract program instead of the concrete one. Consider the program Prog ${ }^{A}$ obtained by replacing every basic predicate $o p\left(X, X^{\prime}\right)$ on the concrete variables by a predicate $o p^{A}\left(X^{A}, X^{A^{\prime}}\right)$ on the abstract variables is a $Q$-abstraction of $\operatorname{Prog}$ if, instead of (1), for every basic operation

$$
\exists X \exists X^{\prime} \cdot \varrho\left(X, X^{A}\right) \wedge \varrho\left(X^{\prime}, X^{A^{\prime}}\right) \wedge o p\left(X, X^{\prime}\right) \Rightarrow o p^{A}\left(X^{A}, X^{A^{\prime}}\right)
$$

holds. If the expressions in Prog are negation free (as in our buffer), then $\operatorname{Prog}^{A}$ is in fact a $Q$-abstraction of Prog. The definition of abstract predicates $o p^{A}$ is the only part of our verification method which cannot be fully automatized. But as we will see, we only need a restricted number of such abstract operations in order to verify a whole class of programs. For example, in the domain of protocol verification, the used data structures are "messages" on which no operations are carried out, "memories" or "registers" in which data can be stored, integers which are mostly used as counters and "buffers" with the usual operations add, tail, first,..., as in our examples. In [CGL91] a similar method is proposed.

Example 2 An abstract lossy buffer. To illustrate the idea, consider again the buffer of Example 1. In order to show that the buffer has the property of "order preservation" (see Example 3), it is sufficient to show that the order of any pair of elements $\left(e_{1}, e_{2}\right) \in$ elem $\times$ elem is preserved. All the information we need about the content of the buffer $B$ is, if and in which order, it contains the elements $\mathbf{e}_{1}$ and $\mathbf{e}_{2}$. Furthermore, as each element is supposed to be put into the buffer at most once, we need not distinguish amongst the valuations of $B$ containing $e_{i}$ more than once. Similarly, for the input variable $e$ we only need to distinguish if its value is $\mathbf{e}_{1}, \mathbf{e}_{2}$ or any other value. Concerning the value of $E$ determinating which events push(e) are still allowed, we only need to know if the event push $\left(\mathrm{e}_{1}\right)$, respectively $p u s h\left(\mathrm{e}_{2}\right)$ is still possible or not. This leads us naturally to the abstract domain defined by the abstract variables,

$$
\begin{aligned}
& e_{A}: \text { elem }_{A}^{2}=\{0,1,2\} \\
& E_{A}^{1}, E_{A}^{2}: \text { Bool } \\
& B_{A}: \text { buffer }{ }_{A}^{2}=\left\{\epsilon, \mathbf{e}_{1}, \mathbf{e}_{2}, \mathbf{e}_{1} \bullet \mathbf{e}_{2}, \mathbf{e}_{2} \bullet \mathbf{e}_{1}, \perp\right\}
\end{aligned}
$$

and the following abstraction relation $\varrho^{2}$ defining the correspondence between the concrete and the abstract variables

$$
\begin{aligned}
\varrho^{2}\left(e, E, B, e_{A},\left(E_{A}^{1}, E_{A}^{2}\right), B_{A}\right)= & \varrho_{\text {elem }}^{2}\left(e, e_{A}\right) \wedge \varrho_{s e t_{-o f \_e l e m}^{2}\left(E,\left(E_{A}^{1}, E_{A}^{2}\right)\right) \wedge} \\
& \varrho_{b u f f e r}^{2}\left(B, B_{A}\right)
\end{aligned}
$$

where for $e:$ elem and $e_{A}:$ elem $_{A}^{2}$

$$
\begin{aligned}
\varrho_{e l e m}^{2}\left(e, e_{A}\right)= & \left(\left(e_{A}=0\right) \equiv\left(e \notin\left\{\mathrm{e}_{1}, \mathrm{e}_{2}\right\}\right)\right) \wedge \\
& \left(\left(e_{A}=1\right) \equiv\left(e=\mathrm{e}_{1}\right)\right) \wedge \\
& \left(\left(e_{A}=2\right) \equiv\left(e=\mathrm{e}_{2}\right)\right)
\end{aligned}
$$

for $E$ : set of elem and $E_{A}^{1}, E_{A}^{2}: B o o l, E_{A}^{i}$ expresses that $\mathbf{e}_{i}$ has not occurred yet: 


$$
\begin{aligned}
\varrho_{\text {set_of_elem }}^{2}\left(E, E_{A}^{1}, E_{A}^{2}\right)= & \left(\left(E_{A}^{1}=\exists E^{\prime} . \operatorname{allowed}\left(\mathbf{e}_{1}, E, E^{\prime}\right) \wedge\right.\right. \\
& \left(\left(E_{A}^{2}=\exists E^{\prime} . \operatorname{allowed}\left(\mathbf{e}_{2}, E, E^{\prime}\right)\right.\right.
\end{aligned}
$$

and for $B: b u f f e r$ of elem and $B_{A}:$ buffer ${ }_{A}^{2}$

$$
\begin{aligned}
& \varrho_{b u f f e r}^{2}\left(B, B_{A}\right)=
\end{aligned}
$$

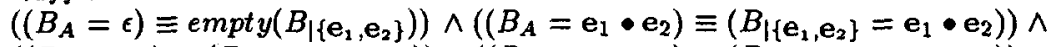

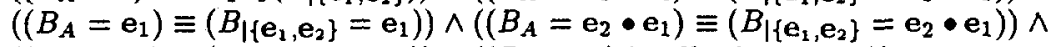

$$
\begin{aligned}
& \left.\left(\left(B_{A}=\mathbf{e}_{2}\right) \equiv\left(B_{\left\{\left\{\mathbf{e}_{1}, e_{2}\right\}\right.}=\mathbf{e}_{2}\right)\right) \wedge\left(\left(B_{A}=\perp\right) \text { in all other cases }\right)\right)
\end{aligned}
$$

where $B_{\left\{\mathbf{e}_{1}, \mathbf{e}_{2}\right\}}$ is the buffer $B$ restricted to the elements $\mathbf{e}_{1}$ and $\mathbf{e}_{2}$. In order to construct an abstract program, we have to define abstract predicates for all the basic predicates used in the concrete buffer program, such as allowed, add, tail, unch, etc.

In the case that every abstract variable is related to a single concrete variable, the abstract predicate associated with $\operatorname{unch}(v)$ is obviously $\operatorname{unch}\left(v_{A}\right)$ for any abstract variable $v_{A}$ related to $v$. The following abstract predicates satisfy the condition (2).

$$
\begin{aligned}
\text { allowed }_{A}^{2}\left(e_{A},\left(E_{A}^{1}, E_{A}^{2}\right),\left(E_{A}^{1^{\prime}}, E_{A}^{2^{\prime}}\right)\right)= & \left(E_{A}^{1^{\prime}} \equiv E_{A}^{1}\right) \wedge\left(E_{A}^{2^{\prime}} \equiv E_{A}^{2}\right) \wedge\left(e_{A}=0\right) \vee \\
& \left.\left(E_{A}^{1} \wedge \neg E_{A}^{1^{\prime}}\right) \wedge\left(E_{A}^{2} \equiv E_{A}^{2^{\prime}}\right)\right) \wedge\left(e_{A}=1\right) \vee \\
& \left.\left.\left(E_{A}^{1} \equiv E_{A}^{1^{\prime}}\right) \wedge\left(E_{A}^{2} \wedge \neg E_{A}^{2 \prime}\right)\right)\right) \wedge\left(e_{A}=2\right)
\end{aligned}
$$

$$
\begin{array}{rr}
\operatorname{add}_{A}^{2}\left(B_{A}, e_{A}, B_{A}^{\prime}\right)=\left(B_{A}=B_{A}^{\prime}\right) & \wedge\left(e_{A}=0\right) \vee \\
& \left(B_{A} \in\left\{\epsilon, \mathbf{e}_{2}\right\}\right) \wedge\left(B_{A}^{\prime}=\mathbf{e}_{1} \bullet B_{A}\right) \vee\left(B_{A} \notin\left\{\epsilon, \mathbf{e}_{2}\right\}\right) \wedge\left(B_{A}^{\prime}=\perp\right) \wedge\left(e_{A}=1\right) \vee \\
& \left.\left(B_{A} \in\left\{\epsilon, \mathbf{e}_{1}\right\}\right) \wedge\left(B_{A}^{\prime}=\mathbf{e}_{2} \bullet B_{A}\right) \vee\left(B_{A} \notin\left\{\epsilon, \mathbf{e}_{1}\right\}\right) \wedge\left(B_{A}^{\prime}=\perp\right)\right) \wedge\left(e_{A}=2\right)
\end{array}
$$

$$
\begin{aligned}
\operatorname{tail}_{A}^{2}\left(B_{A}, e_{A}, B_{A}^{\prime}\right)= & \wedge\left(B_{A}=B_{A}^{\prime}\right) \\
& \left(\left(B_{A} \in\left\{\mathbf{e}_{1}, \mathbf{e}_{1} \bullet \mathbf{e}_{2}\right\}\right) \Rightarrow\left(B_{A}=B_{A}^{\prime} \bullet \mathbf{e}_{1}\right)\right) \wedge\left(e_{A}=1\right) \vee \\
& \left(\left(B_{A} \in\left\{\mathbf{e}_{2}, \mathbf{e}_{2} \bullet e_{1}\right\}\right) \Rightarrow\left(B_{A}=B_{A}^{\prime} \bullet \mathbf{e}_{2}\right)\right) \wedge\left(e_{A}=2\right)
\end{aligned}
$$

$$
\begin{aligned}
\operatorname{empty} y_{A}^{2}\left(B_{A}\right)= & \left(B_{A}=\epsilon\right) \\
\text { first }_{A}^{2}\left(B_{A}, e_{A}\right)= & \left(e_{A}=0\right) \vee \\
& \left(B_{A} \in\left\{\mathbf{e}_{1}, \mathbf{e}_{1} \bullet e_{2}, \perp\right\}\right) \wedge\left(\epsilon_{A}=1\right) \vee \\
& \left(B_{A} \in\left\{\mathbf{e}_{2}, \perp\right\}\right) \wedge\left(e_{A}=2\right)
\end{aligned}
$$

tail is an example of a predicate defining a function on the concrete domain, but which is nondeterministic on the given abstract domain; $\operatorname{tail}_{A}^{2}\left(\perp, 1, B_{A}^{\prime}\right)$ necessarily evaluates to true for any value of $B_{A}^{\prime}$ (the value of the buffer in the next state).

Using these abstract predicates, the definition of a program representing a $\varrho$-abstraction of the buffer program becomes trivial. We just replace variables by corresponding abstract variables and every occurrence of a predicate by corresponding abstract one. The resulting abstract program looks almost as the concrete program but defines a very small finite transition system.

The useful abstractions are often obtained by using this kind of abstract domains. Here, we gave in detail the more complicated abstraction of a buffer particularizing two different data elements. But often, it is sufficient to particularize in the same way a single data element. The corresponding abstraction relations $\varrho_{\text {elem }}^{1}, \varrho_{\text {set_of_elem }}^{1}, \varrho_{b u f f e r}^{1}$ and abstract predicates allowed $\boldsymbol{A}_{A}^{1}, a d d_{A}^{1}, \operatorname{tail}_{A}^{1}, \ldots$ can be defined by simplifying the above definitions in an obvious manner. For the verification of the cache memory we use also existential abstractions of buffers. The corresponding abstract predicates add $d^{e x}\left(e_{A}\right), \operatorname{tail}^{e x}\left(e_{A}\right), \ldots$ necessarily evaluate to true if $e_{A}$ is an allowed value of the existentially abstracted buffer. 


\subsection{Temporal Logic}

It remains to recall the definition of temporal logic. Here we restrict ourselves to subsets of CTL* [EH83] for the expression of properties. The preservation results in [LGS ${ }^{+}$92] are given for subsets of the more powerful branching time $\mu$-calculus augmented by past time modalities.

Definition 2. $\mathrm{CTL}^{*}$ is the set of state formulas given by the following definition.

1. Let $\mathcal{P}$ be a set of atomic (a) state respectively (b) path formulas.

2. If $\phi$ and $\psi$ are (a) state respectively (b) path formulas then $\phi \wedge \psi, \phi \vee \psi$ and $\neg \phi$ are (a) state respectively (b) path formulas.

3. If $\phi$ is a path formula then $\mathbf{A} \phi$ and $\mathbf{E} \phi$ are state formulas.

4. If $\phi$ and $\psi$ are (a) state or (b) path formulas then $\mathbf{X} \phi, \phi \mathcal{U} \psi$ and $\phi \mathcal{W} \psi$ are path formulas.

As usual, we also use the abbreviations $\mathbf{F} \phi$ denoting true $\mathcal{U} \phi$ and $G \phi$ denoting $\phi \mathcal{W}$ false.

$U$ is a strong and $\mathcal{W}$ a weak "until" operator, a sequence satisfies $\phi \mathcal{W} \psi$ if $\phi$ holds up to some point in which $\psi$ holds, and $\phi \mathcal{U} \psi$ expresses the same property and moreover the obligation that such a point satisfying $\psi$ exists. That means that $\mathcal{U}$ and $\mathcal{W}$ are duals by inversing the arguments: $\phi \mathcal{W} \psi=\neg(\neg \psi \mathcal{U} \neg \phi)$.

$\forall C^{*} L^{*}$ [SG90] is the subset of CTL* obtained by allowing negations only on atomic formulas and restricting Rule 3 by allowing only the universal path quantifier $\mathbf{A}$.

The semantics of $\mathrm{CTL}^{*}$ is defined over Kripke structures of the form $M=(S, \mathcal{I})$ where $S=(Q, R)$ is a transition system and $\mathcal{I}$ is a interpretation function mapping elements of $\mathcal{P}$ into sets of states of $S$.

Definition 3. A path in a transition system $S$ is an infinite sequence $\pi=q_{0} q_{1} \ldots$ such that for every $i \in \mathcal{N} . R\left(q_{i}, q_{i+1}\right)$. We denote by $\pi_{n}$ the $n$th state of path $\pi$ and by $\pi^{n}$ the sub-path of $\pi$ starting in its $n$th state.

Definition 4. Let be $M=(S, \mathcal{I})$ a Kripke structure, $q \in Q$ and $\pi$ a path of $M$. Then the satisfaction $\left(\vDash_{M}\right)$ of $\mathrm{CTL}^{*}$ formulas on $M$ is defined inductively as follows.

1. Let be $p \in \mathcal{P}$. Then $q \vDash_{M} p$ iff $q \in \mathcal{I}(p)$ and $\pi \vDash_{M} p$ iff $\pi_{0} \in \mathcal{I}(p)$.

2. Let $\phi$ and $\psi$ be (a) state respectively (b) path formulas. Then,

(a) $q \vDash_{M} \neg \phi$ iff $q k_{M} \phi, q \vDash_{M} \phi \wedge \psi$ iff $q \vDash_{M} \phi$ and $q \vDash_{M} \psi, q \vDash_{M} \phi \vee \psi$ iff $q \vDash_{M} \phi$ or $q \vDash_{M} \psi$.

(b) analogous by replacing $q$ by $\pi$

3. Let $\phi$ be a path formula. Then,

$q \vDash_{M}$ A $\phi$ iff for every path $\pi$ starting in $q, \pi \vDash_{M} \phi$ and

$q \vDash_{M} \mathbf{E} \phi$ iff there exists a path $\pi$ starting in $q$ such that $\pi \vDash_{M} \phi$.

4. Let $\phi$ and $\psi$ be (a) state respectively (b) path formulas. Then,

(a) $\pi \vDash_{M} \mathbf{X} \phi$ iff $\pi_{1} \vDash_{M} \phi$,

$\pi \vDash_{M} \phi \mathcal{U} \psi$ iff there exists $n \in \mathcal{N}$ such that $\pi_{n} \vDash_{M} \psi$ and $\forall k<n . \pi_{k} \vDash_{M} \phi$,

$\pi \vDash_{M} \phi \mathcal{W} \psi$ iff for all $n \in \mathcal{N} .\left(\left(\forall k<n . \pi_{k} \vDash_{M} \neg \psi\right)\right.$ implies $\left.\pi_{n} \vDash_{M} \phi\right)$.

(b) the same definition obtained by replacing in (a) all states $\pi_{i}$ by subsequences $\pi^{i}$.

We say that $M \vDash \phi$ iff $q \vDash_{M} \phi$ for all states of $M$.

From the more general results given in [LGS+92] we obtain the following proposition concerning preservation of properties of $\forall \mathrm{CTL}^{*}$. 
Proposition 5 Preservation of $\forall \mathrm{CTL}^{*}$. Let Prog be a program, $\varrho$ a total relation from the domain of Prog into some abstract domain, and Prog $\operatorname{la}_{A}$ a-abstraction of Prog. Then, for any $\phi \in \forall \mathrm{CTL}^{*}, \mathcal{P}$ the set of atomic formulas occurring in $\phi$ and $\mathcal{I}$ an interpretation function mapping $\mathcal{P}$ into sets of states of $S_{P r o g}$, we have

$$
\begin{aligned}
& \operatorname{Im}\left[\varrho^{-1}\right] \circ \operatorname{Im}[\varrho] \circ \mathcal{I}(p) \subseteq \mathcal{I}(p) \quad\left(^{*}\right) \text { for all } p \in \mathcal{P} \text { occurring positive in } \phi \\
& \text { implies } \\
& \left(S_{P_{\text {rog }}}, \operatorname{Im}[\varrho] \circ \mathcal{I}\right) \vDash \phi \quad \Rightarrow \quad\left(S_{\text {Prog }}, \mathcal{I}\right) \vDash \phi
\end{aligned}
$$

where $\operatorname{Im}[\varrho]$ is the image function of $\varrho$. Condition $\left(^{*}\right)$ is called consistency of $\varrho$ with $\mathcal{I}(p)$.

This proposition expresses that, if $\phi \in \forall \mathrm{CTL}^{*}$ holds on a $\varrho$-abstraction of the program Prog by translating the interpretations of all atomic propositions occurring in the formula by $\operatorname{Im}(\varrho)$ into predicates on the abstract domain, and if all these predicates are consistent with $\varrho$, then we can deduce that $\phi$ holds on Prog. Consistency is not needed for predicates that occur only negated in $\phi$ as $\left.\operatorname{Im}\left[\varrho^{-1}\right] \overline{(m[\varrho](\mathcal{I}(p))}\right) \subseteq \overline{\mathcal{I}(p)}$. We conclude that, if $\phi$ holds on $\operatorname{Prog}_{A}$ using the abstract interpretation of $\neg p(\overline{\operatorname{Im}[\varrho](\mathcal{I}(p))})$, then a stronger property than $\phi$ using the concrete interpretation of $\neg p(\overline{\mathcal{I}(p)})$ holds on Prog. In particular, for the verification of a formula of the form init $\Rightarrow \phi$, init need not to be consistent with $\varrho$.

Example 3. The property of order preservation can be expressed by the following set of formulas on the set of "observable" atomic predicates

$$
\mathcal{P}=\{\text { init, enable }(p u s h(e)), \text { after }(p u s h(e)), \text { enable }(p o p(e)), \text { after }(p o p(e)), \ldots\},
$$

where enable $(\ell)$ is interpreted as the set of states in which event $\ell$ is possible, and after $(\ell)$ those in which $\ell$ has just occurred - after $(\ell)$ becomes expressible by adding an explicit boolean program variable after $\ell$ which is true exactly after any event $\ell$.

$$
\begin{array}{r}
\forall e^{\prime}, e \in \text { elem : init } \Rightarrow \mathbf{A}\left(\left[\neg \text { after }(\text { push }(e)) \text { Wafter }\left(p u s h\left(e^{\prime}\right)\right)\right] \Rightarrow\right. \\
\left.\left[\neg \text { enable }(\operatorname{pop}(e)) \text { Wafter }\left(\operatorname{pop}\left(e^{\prime}\right)\right)\right]\right)
\end{array}
$$

These formulas can be transformed into $\forall C T L^{*}$ formulas in which only the predicates after $(p u s h(e))$ and after $\left(p o p\left(e^{\prime}\right)\right)$ occur non negated. In order to verify that the concrete buffer program has the property of order preservation, it is sufficient to verify the formula obtained by instanciating $\mathbf{e}_{1}$ for $e$ and $e_{2}$ for $e^{\prime}$ on the abstract program defined in Example 2. In fact, as $e_{1}$ and $e_{2}$ represent an arbitrary pair of data values, this verification of a single representative of the set of formulas is sufficient. It is easy to obtain the consistency of predicates of the form after $(\ell)$ by not abstracting the variable after $\ell$. In the sequel we suppose, without mentioning it explicitly, that for every predicate after $(\ell)$ occurring in the considered formula such a variable is defined.

\section{Verification of a distributed cache memory}

\subsection{Concrete and abstract specification of a sequential consistent memory}

Now we use this verification method for the verification of a particular distributed cache memory which has been presented in [ABM93] and verified using different methods in [JPR93, DGJ+93]. The cache memory is a system of the form $P_{1}\left\|P_{2} \ldots\right\| P_{n}$ where each process $P_{i}$ is defined as in Figure 3.1. the predicates add, tail, first and empty are as in the Example 1 and update is defined by update $\left(m,(a, d), m^{\prime}\right) \equiv\left(m^{\prime}[a]=d\right) \wedge\left(\forall b:\right.$ address. $\left.\left(b \neq a \Rightarrow m^{\prime}[b]=m[b]\right)\right)$. 


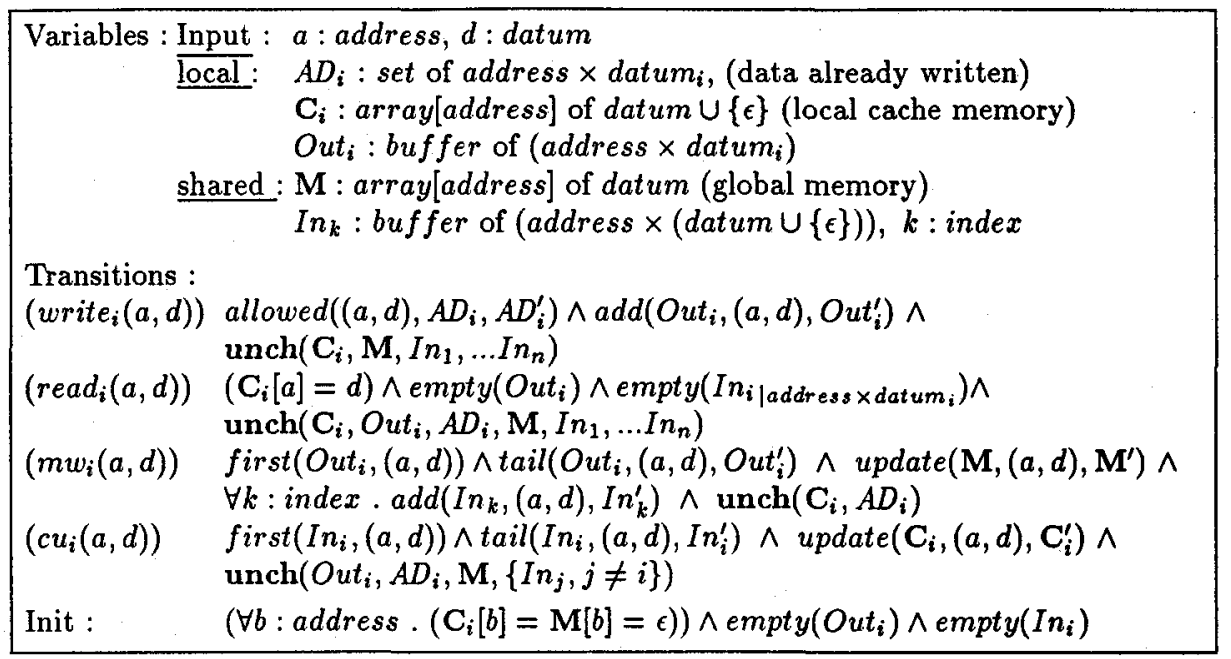

Fig. 1. A distributed cache memory system

The event write $_{i}(a, d)$ does not have any immediate effect neither on the local nor on the central memory, but pushes the pair $(a, d)$ into the local buffer Out $t_{i}$; from there it is by the internal event memory write $_{i}(a, d)$ written into the central memory and dispatched into all buffers $I n_{i}$; the internal event cashupdate ${ }_{i}(a, d)$ takes the first element $(a, d)$ out of $I n_{i}$ and writes datum $d$ into address $a$ of the local cache memory $\mathbf{C}_{i}$. The event $\operatorname{read}_{i}(a, d)$ is possible only if $\mathrm{C}_{i}[a]=d$ and no local write event is pending, i. e., if empty $\left(\right.$ Out $\left._{i}\right) \wedge \operatorname{empty}\left(\right.$ In $\left._{i \text { |address } \times \text { datum }}\right)$. The only difference between our system and the one used in [JPR93] concerns the fact that each pair $(a, d)$ can be the parameter of at most one event write. The way we obtain this, is by defining the type datum by datum $=\bigcup_{i}$ datum $_{i}$, such that each process "signs" the data it writes, and by using in each process a variable $A D_{i}$ of type set of address $\times$ datum $_{i}$ which stores the information if the event write $_{i}(a, d)$ has already occurred or not, as in the example of the buffer.

The abstract specification that the system must verify is sequential consistency [Lam79], which originally is given in the form of an abstract program. In order to apply our method, we give the abstract specification in terms of a set of properties. Under the assumption that every pair of the form $(a, d)$ can occur at most once as the parameter of some write event, sequential consistency can be characterized by the following set of properties expressed in terms of observable events:

Safety properties characterizing a sequential consistent memory:

(S1) $\forall(a, d),\left(a^{\prime}, d^{\prime}\right):$ address $\times$ datum, $j, i:$ index such that $(a, d) \neq\left(a^{\prime}, d^{\prime}\right)$

init $\Rightarrow \mathbf{A}\left(\left[\neg\right.\right.$ after $\left(\right.$ write $\left._{j}(a, d)\right)$ Wafter $\left(\right.$ write $\left.\left._{j}\left(a^{\prime}, d^{\prime}\right)\right)\right] \Rightarrow$

$\left[\neg\right.$ enable $\left(\operatorname{read}_{i}(a, d)\right) \mathcal{W}\left(\right.$ enable $\left(\operatorname{read}_{i}\left(a^{\prime}, d^{\prime}\right)\right) \vee$

$$
\left.\left.\left.A G\left(\neg \text { enable }\left(\operatorname{read}_{i}\left(a^{\prime}, d^{\prime}\right)\right)\right)\right)\right]\right)
$$

(S2) $\forall(a, d):$ address $\times$ datum, $i$ : index 


$$
\text { init } \Rightarrow \mathbf{A}\left(\neg \text { enable }\left(\operatorname{read}_{i}(a, d)\right) \mathcal{W} \bigvee_{j: \text { index }} \operatorname{after}_{\left.\left(\text {write }_{j}(a, d)\right)\right)}\right.
$$

(S3) $\forall(a, d):$ address $\times$ datum, $i$ : index

init $\Rightarrow \mathbf{A G}\left(\right.$ after $\left(\right.$ write $\left._{i}(a, d)\right) \Rightarrow$

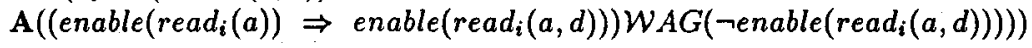

(S4) $\forall(a, d),\left(a, d^{\prime}\right)$ : address $\times$ datum, $i_{1}, i_{2}$ : index such that $d \neq d^{\prime}$

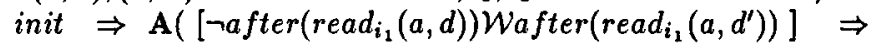
$\left[\neg\right.$ enable $\left.\left.\left(\operatorname{read}_{i_{2}}(a, d)\right) \mathcal{W} A G\left(\neg \operatorname{enable}\left(\operatorname{read}_{i_{2}}\left(a, d^{\prime}\right)\right)\right)\right]\right)$

(S1) expresses that in every execution sequence the subsequence of read $_{i}$ events respects

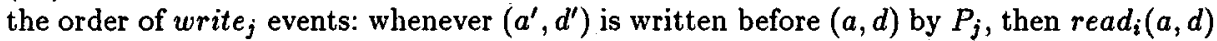
is not enabled before either $\operatorname{read}_{i}\left(a^{\prime}, d^{\prime}\right)$ has already been enabled or is never enabled again (the second clause is necessary because $\operatorname{read}_{i}\left(a^{\prime}, d^{\prime}\right)$ may never be enabled in some computation sequences). (S2) expresses that every readi $(a, d)$ event is preceded (caused) by some write $_{j}(a, d)$ event. This is slightly stronger than sequential consistency which may allow write $j(a, d)$ to occur after $\operatorname{read}_{i}(a, d)$. (S3) expresses the fact that read $d_{i}$ and write $_{i}$ events od the same process $P_{i}$ must be consistent with a central memory, i. e., after a write $_{i}(a, d), P_{i}$ can read nothing different from $d$ in address a until $\operatorname{read}_{i}(a, d)$ is never enabled again. (S4) expresses analogously to (S1) the fact that read events concerning the same memory cell must be consistent in all processes. All these formulas can be translated into $\forall \mathrm{CTL}^{*}$ formulas.

\subsection{Verification of the cache memory}

We verify each parameterized set of formulas on a different abstract program. Our aim is not to find the smallest abstract program that can be used for the verification of each formula, but we want to apply, whenever possible, the already predefined abstractions in order to show that the application of the method is simple and can be done systematically. The cache memory uses the data types and operations of the buffer of Example 1; it uses also a data type "memory" = array[address] of datum. As for buffers, we use three different types of abstractions of a variable $\mathrm{X}$ of type memory depending on the formula to be verified: we may

- completely forget about it (we do this always for the central memory $\mathbf{M}$ )

- keep information about a single pair (a,d) by taking an abstract boolean variable $X_{A}$ and an abstraction relation $\varrho_{\text {memory }}^{1}\left(X, X_{A}\right)=X_{A} \equiv(X[\mathbf{a}]=\mathbf{d})$.

- keep information about two pairs $\left(\mathbf{a}_{1}, \mathbf{d}_{1}\right)$ and $\left(\mathbf{a}_{2}, \mathbf{d}_{2}\right)$ by taking two abstract boolean variables $X_{A}^{1}$ and $X_{A}^{2}$ and an analogous abstraction relation $\varrho_{\text {memory }}^{2}\left(X, X_{A}^{1}, X_{A}^{2}\right)$.

Suppose the type elem to be address $\times$ datum and take an abstract variable $e_{A}$ of type elem ${ }_{A}^{1}=\{0,1\}$ already used in the buffer example and the abstraction relation

$\varrho_{e l e m}^{1}\left((a, d), e_{A}\right)=\left(e_{A}=0\right) \wedge((a, d) \neq(\mathbf{a}, \mathbf{d})) \vee\left(e_{A}=1\right) \wedge((a, d)=(\mathbf{a}, \mathbf{d}))$,

exactly as in Example 2 ; then, it is easy to define an abstract predicate update $e_{A}^{1}$ by

$$
\text { update }{ }_{A}^{1}\left(X_{A}, e_{A}, X_{A}^{\prime}\right)=\left(e_{A}=0\right) \wedge\left(X_{A}^{\prime} \Rightarrow X_{A}\right) \vee\left(e_{A}=1\right) \wedge X_{A}^{\prime}
$$

expressing that if $(a, d) \neq(\mathbf{a}, \mathbf{d}), X[\mathbf{a}]=\mathbf{d}$ is only possible in the next state if already in the present state $X[\mathbf{a}]=\mathbf{d}$, and if $(a, d)=(\mathbf{a}, \mathbf{d})$, then in the next state $X[\mathbf{a}]=\mathbf{d}$, independently of the value of $X[\mathrm{a}]$ in the present state.

Using these definitions (and analogous ones with superscripts ${ }^{e x}$ and ${ }^{2}$ ) and those already given in Example 1, the definition of appropriate abstract finite state programs of the cache memory becomes simple. 
Abstract programs for property (S3): Each instance of property (S3) involves only events of a single process $P_{i}$. However, even if we succeed to verify it on $P_{i}$ we can not deduce their satisfaction on the composed system. In fact, if we replace all processes different from $P_{i}$ by the process "Chaos", (S3) does not hold any more on the composed abstract program. We use here another approach to compositionality: by Proposition 1, we can abstract each $P_{j}$ individually and build a global model by composing these small abstract programs. We choose the abstraction relation for all $P_{j}$ with $j \neq i$ in such a way that shared variables are abstracted in the same way as in $P_{i}$ and we forget about all local variables; this has as effect to avoid adding certain changes of shared variables which are not allowed by the concrete programs $P_{j}$.

Intuitively, (S3) expresses that as soon as write $e_{i}(\mathbf{a}, \mathrm{d})$ has occurred, only $\mathbf{d}$ may be read by $P_{i}$ on address a until $\mathrm{d}$ has been put into $C_{i}$ and afterwards been replaced by some other value; that means that we are interested in observing what happens on the buffers Out $t_{i}$ and $I n_{i}$ and the cache $C_{i}$. The actions $\left(m w_{j}\right)$ should not disturb the behaviour of $P_{i}$ observed by (S3) because they cannot push $(\mathrm{a}, \mathrm{d})$ into $I n_{i}$. This leads naturally to the following abstraction relation for $P_{i}$ :

$$
\begin{aligned}
& \varrho_{i}^{S 3}\left((a, d), A D_{i}, \mathbf{C}_{i}, O u t_{i}, \mathbf{M}, I n_{1}, \ldots I n_{n}, e_{A}, E_{A}, \mathbf{C}_{i A}, O u t_{i A}, I n_{i A}\right)= \\
& \varrho_{\text {elem }}^{1}\left((a, d), e_{A}\right) \wedge \quad \wedge \quad \varrho_{\text {set_of_elem }}^{1}\left(A D_{i}, E_{A}\right) \wedge \\
& \varrho_{m e m o r y}^{1}\left(\mathbf{C}_{i}, \mathbf{C}_{i A}\right) \quad \wedge \quad \varrho_{b u f f e r}^{1}\left(\text { Out }_{i}, \text { Out }_{i A}\right) \wedge \\
& \varrho_{b u f f e r}^{1}\left(I n_{i}, I n_{i A}\right)
\end{aligned}
$$

and for $P_{j}, j \neq i$ we use the same abstraction as in $\varrho_{i}$ for the shared variables and forget about all local variables

$$
\begin{aligned}
& \varrho_{j}^{S 3}\left((a, d), A D_{i}, \mathbf{C}_{i}, O u t_{i}, \mathbf{M}, I n_{1}, \ldots, I n_{n}, e_{A}, I n_{i A}\right)= \\
& \varrho_{\text {elem }}^{1}\left((a, d), e_{A}\right) \wedge \quad \varrho_{b u f f e r}^{1}\left(I n_{i}, I n_{i A}\right)
\end{aligned}
$$

from which we obtain by replacing concrete by corresponding abstract predicates as defined before, the following abstract program $P_{i}^{A}$ for index $i$,

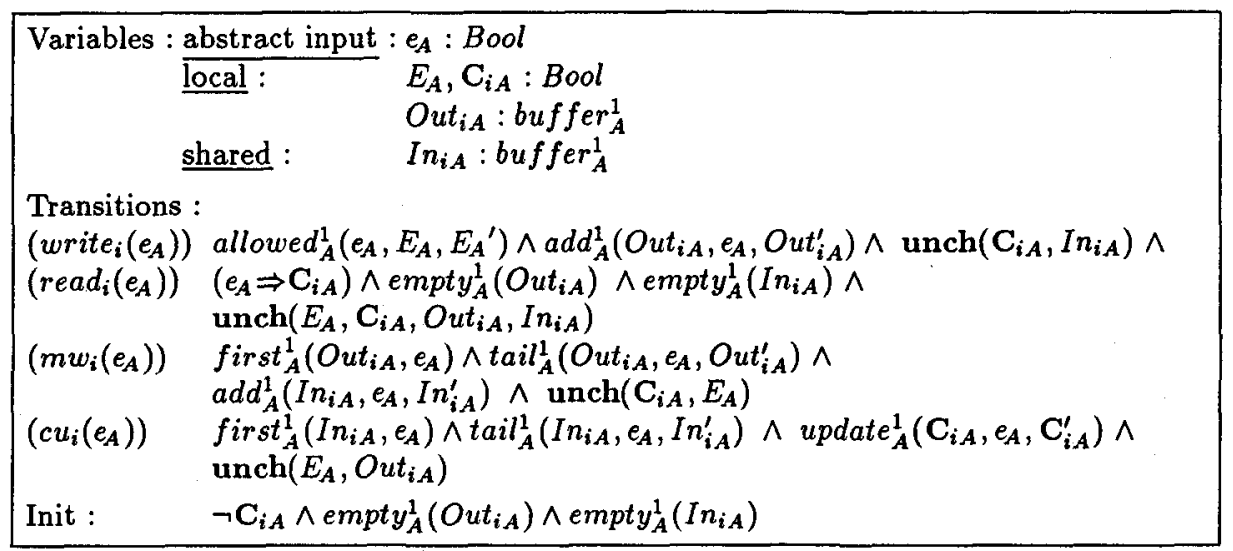

and $P_{j}^{A}$ for all indices different from $i$, 


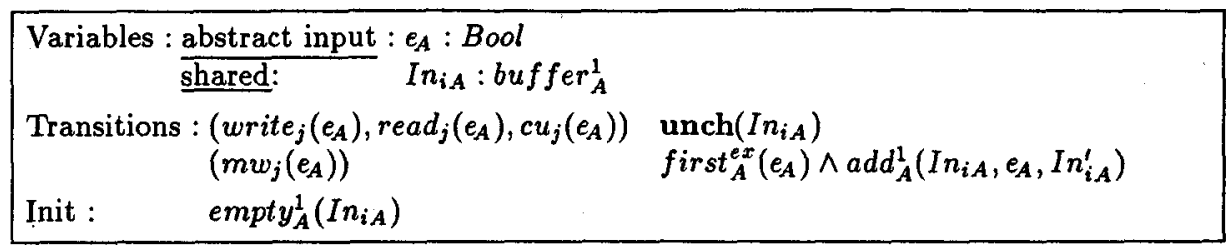

in which we have already eliminated all abstract operations that are always true, such as $a d d_{A}^{e x}$, update $e_{A}^{e x}, \ldots .$. Notice that the event $\left(m w_{j}(\right.$ true $\left.)\right)$ is never executed as first ${ }_{A}^{e x}($ true $)=$ false because the buffer Out $_{j}$ cannot contain the pair $(\mathbf{a}, \mathrm{d})$ as $\mathrm{d} \in$ datum $_{i}$. Notice also that the size of the composed system $P_{1}^{A}\|\ldots\| P_{i}^{A}\|\ldots\| P_{n}^{A}$ is the same, whatever the number of composed programs is, as for all $j \neq i$, the programs $P_{j}^{A}$ are identical and $P \| P$ and $P$ represent the same transition system.

Abstract programs for property (S2): Property (S2) expresses the fact that any event $\operatorname{read}_{i}(\mathbf{a}, \mathrm{d})$ is preceded by an event write $_{j}(\mathbf{a}, \mathrm{d})$ for some $j$. Thus, we are interested in observing the buffers $O u t_{j}$ and $I n_{i}$ and the cache $C_{i}$. This leads to similar abstraction relations as for the verification of (S3), except that we do not need the unicity of the write events and can forget about $A D_{i}$ but we need abstract buffers $O u t_{j A}$ for all $j$. Thus, all abstraction relations $\varrho_{j}^{S 2}$ are the same:

$$
\begin{aligned}
& \varrho_{j}^{S 2}\left(A, D, A D_{j}, \mathbf{C}_{j}, \text { Out }_{j}, \mathbf{M}, I n_{1}, \ldots, I n_{n}, e_{A}, \text { Out }_{j A}, I n_{i A}\right)= \\
& \varrho_{\text {elem }}^{1}\left((A, D), e_{A}\right) \wedge \wedge \quad \varrho_{b u f f e r}^{1}\left(\text { Out }_{j}, \text { Out }_{j A}\right) \wedge \\
& \varrho_{b u f f e r}^{1}\left(I n_{i}, I n_{i A}\right)
\end{aligned}
$$

For this abstraction, the size of the obtained global abstract transition system does depend on the number $n$ of processes as we have defined $n$ abstract variables $O u t_{j A}$. In order to obtain an abstract transition system such that its size is independent of $n$, we can define - instead of the set of local abstract buffers $O u t_{j A}$ - a single global abstract buffer $O u t_{A}$ defined by a relation of the form

$$
\varrho_{\text {buffer }}^{1, g l o b}\left(\bigcup_{j: \text { index }} \text { Out }_{j}, \text { Out }_{A}\right)
$$

which obliges however to redefine the abstract operations $\operatorname{add}_{A}, \operatorname{tail}_{A}, \ldots$

Abstract programs for properties (S1) and (S4): For the verification of (S1) we need to observe events with two different parameters $\left(\mathbf{a}_{1}, \mathbf{d}_{1}\right)$ and $\left(\mathbf{a}_{2}, \mathbf{d}_{2}\right)$, such that $\mathbf{d}_{1}, \mathbf{d}_{2} \in$ datum $_{j}$; thus, we use the abstraction relations with superscript ${ }^{2}$ as for the verification of order preservation in the preceding section. We define abstract variables $E_{A}^{1}, E_{A}^{2}$ (in $P_{j}^{A}$ ) in order to guarantee uniqueness of the observed write $e_{j}$ events, Out ${ }_{j A}$ (in $P_{j}^{A}$ ), $\mathrm{C}_{i_{1}}, \mathrm{C}_{i_{2}}$ (in $P_{i}^{A}$ ) and a global variable $I n_{i_{A} A}$ and use the predefined abstraction relations and corresponding abstract operations.

The resulting global abstract transition system is again independent of the number of process as all the abstract programs with indices different from $i, j$ are identical. We need only to consider the case where the indices $i$ and the $j$ are different, as the property for $i=j$ is implied by (S3).

Property (S4) expresses that the sequences of read events of any two processes $P_{i_{1}}$ and $P_{i_{2}}$ on the same address a are compatible, also when they have been written by two different processes $P_{j_{1}}$ and $P_{j_{2}}$. Thus, for its verification we observe two pairs $\left(\mathbf{a}_{1}, \mathrm{~d}_{1}\right)$ and 
$\left(\mathbf{a}_{2}, \mathbf{d}_{2}\right)$ such that $\mathbf{a}_{1}=\mathbf{a}_{2}=\mathbf{a}$ and $\mathbf{d}_{1} \in$ datum $j_{j_{1}}$ and $\mathbf{d}_{2} \in$ datum $m_{j_{2}}$. Consequently, we need abstract variables $E_{A}^{1}, E_{A}^{2}$ (in $P_{j_{1}}^{A}$ respectively $P_{j_{2}}^{A}$ ), Out $t_{j_{1} A}, O u t_{j_{2} A}$ (in $P_{j_{1}}^{A}$ respectively $P_{j_{2}}^{A}$ ), $\mathbf{C}_{i_{1} 1}, \mathbf{C}_{i_{1} 2}$ (in $P_{i_{1}}^{A}$ ), $\mathbf{C}_{i_{2} 1}, \mathbf{C}_{i_{2} 2}$ (in $P_{i_{2}}^{A}$ ) and global variables $I n_{i_{1} A}$ and $I n_{i_{2} A}$. Here, we have to consider different cases, those where the indices $i_{1}$ and $j_{1}$ (respectively $i_{2}$ and $j_{2}$ ) coincide and those where not.

Now, the verification of the distributed cache memory is almost terminated. The actual construction of global abstract transition systems and the verification of the formulas on them could been done automatically by our tool [GL93b, Loi94]. By Proposition 5, it remains to verify the consistency of the atomic propositions with the used abstraction relations. Properties (S2) and (S4) pose no problem, as in the corresponding $\forall \mathrm{CTL}^{*}$ formulas only predicates of the form after( $\ell$ ) occur non negated. For (S3), in principle the consistency of enable $\left(\operatorname{read}_{i}(\mathbf{a}, \mathbf{d})\right)$ is required; however, it is used only within the predicate $\left(\operatorname{enable}\left(\operatorname{read}_{i}(\mathbf{a})\right) \Rightarrow \operatorname{enable}\left(\operatorname{read}_{i}(\mathbf{a}, \mathbf{d})\right)\right.$ which is equivalent to $\mathbf{C}_{i}[\mathbf{a}]=\mathbf{d}$ and consistent with the abstraction relation used for (S3). For (S1), it is slightly more complicated to show that the consistency of enable $\left(\operatorname{read}_{i_{2}}\left(\mathbf{a}_{2}, \mathbf{d}_{2}\right)\right)$ with the considered abstraction relation $\varrho$ is not needed. The predicate obtained by translating enable $\left(\operatorname{read}_{i_{2}}\left(\mathbf{a}_{2}, \mathbf{d}_{2}\right)\right)$ forth and back by $\varrho$ is $\mathbf{C}_{i_{2}}\left[\mathbf{a}_{2}\right]=\mathbf{d}_{2} \wedge \ldots$ The property obtained from (S1) by replacing enable $\left(\right.$ read $\left._{i_{2}}\left(\mathbf{a}_{2}, \mathbf{d}_{2}\right)\right)$ by this weaker predicate, implies nevertheless (S1) for our particular system, because enable $\left(\operatorname{read}_{i_{2}}\left(\mathbf{a}_{1}\right) \Rightarrow\left(\left(\mathbf{C}_{i}\left[\mathbf{a}_{2}\right]=\mathbf{d}_{2}\right) \Rightarrow\right.\right.$ enable $\left(\right.$ read $\left.\left._{i_{2}}\left(\mathbf{a}_{2}, \mathbf{d}_{2}\right)\right)\right)$ holds. Notice also, that this additional condition is in fact necessary in order to obtain sequential consistency of the given system.

\section{Discussion}

What have we achieved? A first impression could be that our verification of a cache memory looks much like any other handwritten proof. However, it is quite different: starting right from the beginning, it is in fact rather lengthy to define all the abstraction relations and corresponding abstract predicates, even in order to verify some trivial buffer program. However, having done this once, in order to verify the much more complex cache memory system, we only need a few more definitions obtained a long the same line as the already given ones. In fact, there are many examples of systems, for which we have to verify exactly the same type of properties and which use analogous data structures and operations on them, such that the same abstract domains and operations can be used. Thus, we could build a "library" of useful abstract domains and operations in which new definitions can be added when necessary. A similar approach has been followed by P. and R. Cousot and D. Long concerning "standard" abstractions of integers and operations on them.

The fact that for the verification of an individual property a large part of the system can be abstracted existentially is often necessary in order to obtain tractable global models. If the system is too large or the property is "too global" one can often get results by decomposing the property, as this has been proposed, e. g. in [Kur89].

It can also been observed that our verification method is incremental: it is obviously incremental with respect to changes in the abstract specification, like every method based on the fact that abstract specifications are expressed by a set of properties. But also certain changes of the program allow to use the same or at least very similar abstraction relations and abstract operations. That means that exactly the time consuming and difficult part of the verification process need not to be redone. In the case that the obtained abstract 
program is not identical to the previous one, the reconstruction of a model and the verification of the properties on it by means of some model checker poses no problem.

An important point for the use of our method in practice, is the formalisms used for the description of programs. We either need a formalism allowing to express nondeterminism or we have to use more complex abstract domains allowing to represent certain sets of classes of concrete values. For example, LоTоs is a specification formalism for which this method can be applied: all the data types and operations on them are specified separately from the control part by means of some "abstract data type" language. This means that, by coding whatever should be abstracted in the data part, to construct an abstract program consists simply in replacing the original data type definitions by simpler ones, whereas the control part of the program remains completely unchanged.

Acknowledgements: I would like to thank the referees who pointed out that my initial characterization of sequential consistency was not sufficient.

\section{References}

[ABM93] Y. Afek, G. Brown, and M. Meritt. Lazy caching. ACM Transactions on Programming Languages and Systems, 15(1), 1993.

[CC77] P. and R. Cousot. Abstract interpretation: a unified lattice model for static analysis of programs by construction or approximation of fixpoints. In POPL, 1977.

[CGL91] E. Clarke, O. Grumberg, D. Long. Model checking and abstraction. In POPL, 1991.

[DGJ+93] J. Davis, R. Gerth, W. Jannsen, B. Jonsson, S. Katz, G. Lowe, A. Pnueli, and C. Rump. Verifying sequentially consistent memory. Preliminary report, 1993.

[EH83] E. A. Emerson and J. Y. Halpern. 'Sometimes' and 'not never' revisited: On branching versus linear time. In $P O P L, 1983$. also in Journal of ACM, 33:151-178.

[GL93a] S. Graf and C. Loiseaux. Program verification using compositional abstraction. In TAPSOFT 93, joint conference CAAP/FASE. LNCS 668, Springer Verlag, April 1993.

[GL93b] S. Graf and C. Loiseaux. A tool for symbolic program verification and abstraction. In CAV, Heraklion Crete. LNCS 697, Springer Verlag, 1993.

[JPR93] B. Jonsson, A. Pnueli, and C. Rump. Proving refinement using transduction. Technical report, Weizmann Institute, 1993.

[Kur89] R.P. Kurshan. Analysis of discrete event coordination. In REX Workshop on Stepwise Refinement of Distributed Systems, Mook. LNCS 430, Springer Verlag, 1989.

[Lam79] L. Lamport. How to make a multiprocessor that correctly executes multiprocess programs. IEEE Transactions on Computers, C-28:690-691, 1979.

[Lam91] L. Lamport. The temporal logic of actions. Technical Report 79, DEC Systems Research Center, 1991.

[LGS ${ }^{+}$92] C. Loiseaux, S. Graf, J. Sifakis, A. Bouajjani, and S. Bensalem. Property preserving abstractions for the verification of concurrent systems. To appear in Formal Methods in System Design, also in CAV'92.

[Loi94] C. Loiseaux. Vérification symbolique de programmes réactifs à l'aide d'abstractions. Thesis, Verimag, Grenoble, January 1994.

[Lon93] D. E. Long. Model checking, abstraction and compositional verification. Phd thesis, Carnegie Mellon University, July 1993.

[Pnu85] A. Pnueli. In transition from global to modular temporal reasoning about programs. In Logics and Models for Concurrent Systems. NATO, ASI Series F, Vol.13, 1985.

[Pnu86] A. Pnueli. Specification and Development of reactive Systems. In Conference IFIP, Dublin. North-Holland, 1986.

[SG90] G. Shurek and O. Grumberg. The modular framework of computer-aided verification: motivation, solutions and evaluation criteria. In $C A V$, LNCS 531, 1990. 